\title{
Identification and characterization of the thaumatin-like genes in garlic
}

Anisimova O.K. ${ }^{1,2 *}$, Filyushin M.A. ${ }^{2}$

${ }^{1}$ Lomonosov Moscow State University, Moscow, Russia

${ }^{2}$ Research Centre of Biotechnology RAS, Moscow, Russia

*email:lelikanis@yandex.ru

Significant yield losses of crops, including garlic Allium sativum, are associated with fungal infections, in response to which pathogenesis-related proteins (PRs) are synthesized. Thaumatin-like proteins (TLPs), that belong to one of 17 known families of PR proteins - PR5, are involved in plant responses to fungal infections. In this study, thaumatin-like proteins genes were identified and characterized in A. sativum genome, and their expression was determined in response to fungus Fusarium proliferatum infection.

In A. sativum cv. Ershuizao genome (PRJNA606385) 32 sequences encoding thaumatinlike proteins were identified. The sizes of the found genes varied from 615 to $2572 \mathrm{bp}$ and the corresponding amino acid sequences included from 204 to 323 aa. Phylogenetic relationships were established and five subclusters of AsTLPs were identified. The presence of various $c i s$-regulatory elements associated with responses to phytohormones and stress factors was predicted in AsTLPS genes promoter regions. AsTLPs gene expression patterns were determined in the roots, stems, and cloves of two garlic cultivars - Sarmat and Strelets (resistant and susceptible to fusarium rot respectively) in response to $F$. proliferatum infection. In general, the AsTLPS expression showed significantly more intensive changes in response to the infection in cv. Sarmat than in cv. Strelets. For the majority of AsTLPs genes, an increase in expression was found in response to $F$. proliferatum infection. In roots of cv. Sarmat, the expression of AsTLP8, AsTLP24, and AsTLP27 increased by many times in 96 hours after the infection. The least pronounced increase in expression in response to the infection was observed in cloves, which is probably associated with the penetration of the pathogen through the roots, where the response was the highest. For differentially expressed AsTLPs genes, promoter regions (1000 bp) were identified and investigated. The obtained results indicate the active participation of AsTLPs genes in the response to $F$. proliferatum infection and in the resistance formation of garlic plants to fusarium rot.

Acknowledgments: This work was supported by the RFBR grant No. 20-316-70009. 